\title{
FIXED POINT THEOREMS FOR COMPACT ACYCLIC METRIC SPACES
}

\author{
SEHIE PARK
}

(Communicated by Dennis Burke)

\begin{abstract}
Multifunctions $F: X \rightarrow 2^{M}$ are studied where $X \subset M$ is either a compact acyclic finite-dimensional ANR or a compact acyclic lc subspace. Conditions are found for the existence of $v$ such that $d(v, F v)=d(X, F v)$ and, also, for the existence of fixed points.
\end{abstract}

1. Introduction and preliminaries. In [7], McClendon proved variations of Ky Fan's minimax inequality in [5]. His main idea is the weakening of "convex" to "contractible" or "acyclic". He used his results to obtain some variational inequalities and fixed point theorems on a function $f: X \rightarrow(M, d)$ with a condition on the "boundary", where $X \subset M$ is either a compact acyclic finite-dimensional ANR or a compact acyclic lc subspace.

In the present paper, we generalize these fixed point theorems to a multifunction $F: X \rightarrow 2^{M}$ with a more general condition than the one on the "boundary". Actually, we give a number of equivalent formulations of such fixed point theorems in general setting.

We begin with the following two results of McClendon [7, Theorems 3.1 and 3.2].

THEOREM 1.1 [7, THEOREM 3.1]. Suppose that $X$ is a compact acyclic finitedimensional ANR. Suppose $p: X \times X \rightarrow R$ is a function such that

(a) $\{(x, y) \mid p(x, x)>p(y, x)\}$ is open in $X \times X$, and

(b) $\{y \mid p(x, x)>p(y, x)\}$ is contractible or empty for all $x \in X$.

Then there exists a point $v \in X$ such that $p(v, v) \leq p(w, v)$ for all $w \in X$.

THEOREM 1.2 [7, THEOREM 3.2]. Suppose that $X$ is a compact acyclic lc space. Let $p: X \times X \rightarrow R$ be a function such that

(a) $p$ is continuous on the diagonal $\{(x, x) \mid x \in X\}$,

(b) $p$ is l.s.c.,

(c) $p(x, \cdot)$ is u.s.c. for all $x \in X$, and

(d) $\{x \mid p(x, y) \leq s\}$ is acyclic or empty for all $s \in R$ and $y \in X$.

Then there exists a point $v \in X$ such that $p(v, v) \leq p(w, v)$ for all $w \in X$.

REMARK. Due to McClendon's proof of Theorem 2.2 [7] it suffices to assume (d) for $s=p(y, y)-\delta(n)$, all $n$, where $\{\delta(n)\}$ is a sequence of positive reals converging to 0 .

Received by the editors October 28, 1986 and, in revised form, March 23, 1987.

1980 Mathematics Subject Classification (1985 Revision). Primary 55M20; Secondary 54H25, 47H10.

Key words and phrases. Contractible, acyclic, fixed point theorems, ANR, u.s.c., l.s.c., lc space, stationary point, closed-valued multifunction, fixed point property.

Partially supported by a grant from Ministry of Education, 1986-87.

(C) 1988 American Mathematical Society $0002-9939 / 88 \$ 1.00+\$ .25$ per page 
A space is contractible if the identity map is homotopic to a constant, e.g., a nonempty convex set in a topological vector space (t.v.s.). A nonempty space is acyclic if it is connected and its Cech homology (with a fixed coefficient field) is zero in dimensions greater than zero. Note that every contractible space is acyclic, but not conversely.

A multifunction $F: X \rightarrow 2^{Y}$ is continuous if it is u.s.c. and l.s.c. ANR means ANR (metric). For the definition of a lc space, see $[\mathbf{1}, \mathbf{8}, \mathbf{9}]$. Note that an ANR is an lc space and a finite union of compact convex subsets of a locally convex t.v.s. is an lc space. Let us denote $d(y, F x)=\inf \{d(y, z) \mid z \in F x\}$.

We add one more variational inequality.

THEOREM 1.3. Let $X$ be a compact acyclic lc space. Let $p: X \times X \rightarrow R$ be a continuous function such that $\{x \mid p(x, y)=P(y)\}$ is acyclic for all $y \in X$, where $P(y)=\inf \{p(x, y) \mid x \in X\}$. Then there exists a point $v \in X$ such that $p(v, v) \leq p(w, v)$ for all $w \in X$.

ProOF. Define a multifunction $F: X \rightarrow 2^{X}$ by $F(y)=\{x \mid p(x, y)=P(y)\}$ for each $y \in X$. Since $F$ is a closed-graph acyclic-valued multifunction, $F$ has a fixed point $v \in X$ by Begle's result [1], and $v$ is a desired one.

2. Main results. First from Theorem 1.1 we have

THEOREM 2.1. Let $X$ be a compact acyclic finite-dimensional ANR subset of a metric space $(M, d)$, and $F: X \rightarrow 2^{M} \backslash\{\phi\}$ a multifunction satisfying

(a) $\{(x, y) \mid d(x, F x)>d(y, F x)\}$ is open in $X \times X$, and

(b) for each $x \in X,\{y \in X \mid d(x, F x)>d(y, F x)\}$ is contractible or empty.

Then the following equivalent statements hold.

(i) There exists a point $v \in X$ such that

$$
d(v, F v) \leq d(w, F v) \quad \text { for all } w \in X .
$$

(ii) If $T: X \rightarrow 2^{M}$ is a multifunction such that for each $x \in X \backslash T x$ there exists a $y \in X$ such that $d(x, F x)>d(y, F x), T$ has a fixed point $v \in X$, that is, $v \in T v$.

(iii) If $g: X \rightarrow M$ is a function such that for each $x \in X$ with $x \neq g x$, there exists a $y \in X$ such that $d(x, F x)>d(y, F x)$, then $g$ has a fixed point $v \in X$.

(iv) If $T: X \rightarrow 2^{X} \backslash\{\phi\}$ is a multifunction such that $d(x, F x)>d(y, F x)$ holds for any $x \in X$ and $y \in T x \backslash\{x\}$, then $T$ has a stationary point $v \in X$, that is, $\{v\}=T v$.

(v) If $\mathscr{F}$ is a nonempty family of functions $g: X \rightarrow X$ such that $d(x, F x)>$ $d(g x, F x)$ holds for any $x \in X$ with $x \neq g x$, then $\mathscr{F}$ has a common fixed point $v \in X$.

(vi) If $g: X \rightarrow X$ is a function such that $d(x, F x)>d(g x, F x)$ holds for any $x \in X$ with $x \neq g x$, then $g$ has a fixed point $v \in X$.

Proof. (i) Define $p: X \times X \rightarrow R$ by $p(x, y)=d(x, F y)$. Then (i) follows from Theorem 1.1.

(i) $\Rightarrow$ (ii) Suppose $v \notin T v$. Then there exists a $y \in X$ such that $d(v, F v)>$ $d(y, F v)$, which contradicts (i).

(ii) $\Rightarrow$ (iii) Clear.

(iii) $\Rightarrow$ (iv) Suppose $T$ has no stationary point, that is, $T x \backslash\{x\} \neq \varnothing$ for each $x \in X$. Choose a choice function $g$ on $\{T x \backslash\{x\} \mid x \in X\}$. Then $g$ has no fixed 
point. However, for any $x \in X$, we have $x \neq g x$ and there exists a $y \in T x \backslash\{x\}$, say $y=g x$, such that $d(x, F x)>d(y, F x)$. Therefore, by (iii), $g$ has a fixed point, a contradiction.

(iv) $\Rightarrow$ (v) Define a multifunction $T: X \rightarrow 2^{X}$ by $T x:=\{g x \mid g \in \mathscr{F}\} \neq \varnothing$ for all $x \in X$. Since $d(x, F x)>d(g x, F x)$ for any $x \in X$ and any $g \in \mathscr{F}$, by (iv), $T$ has a stationary point $v \in X$, which is a common fixed point of $\mathscr{F}$.

(v) $\Rightarrow$ (vi) Put $\mathscr{F}=\{g\}$.

(vi) $\Rightarrow$ (i) Suppose that for any $x \in X$, there exists a $y \in X$ satisfying $d(x, F x)>$ $d(y, F x)$. Choose $g x$ to be one of such $y$. Then $g: X \rightarrow X$ has no fixed point by its definition. However, $d(x, F x)>d(g x, F x)$ for all $x \in X$. By (vi), $g$ has a fixed point, a contradiction.

This completes our proof.

Define $\partial X=\partial_{M} X=\{y \in X \mid$ there is a $z \in M \backslash X$ with $d(z, y)=d(z, X)\}[\mathbf{7}]$.

COROLLARY 2.1.1. Let $X$ be a compact acyclic finite-dimensional ANR subset of a metric space $M$, and $F: X \rightarrow 2^{M} \backslash\{\phi\}$ continuous compact-valued such that for all $x \in X,\{y \in X \mid d(x, F x)>d(y, F x)\}$ is contractible or empty.

Then (i) (vi) of Theorem 2.1 hold. Further, $F$ has a fixed point if one of the following conditions holds.

(1) For each $x \in X \backslash F x$, there exists a $y \in X$ such that $d(x, F x)>d(y, F x)$.

(2) $F x \cap X \neq \varnothing$ for each $x \in \partial X$.

(3) $F x \cap X \neq \varnothing$ for each $x \in X$.

(4) Fx $\subset X$ for each $x \in X$.

Proof. Since $F$ is continuous, the condition (a) in Theorem 2.1 holds by Theorems 1 and 2 in Berge [2, p. 121]. Therefore, (i) (vi) hold.

(1) By putting $F=T$ in (ii), if condition (1) holds, then $F$ has a fixed point.

(2) We show that (2) $\Rightarrow(1)$. Suppose that there exists an $x \in X \backslash F x$ such that $d(x, F x) \leq d(y, F x)$ holds for all $y \in X$. If $F x \cap X \neq \varnothing$, then $d(y, F x)=0$ for some $y \in F x \cap X$. Therefore, $d(x, F x)=0$, whence $x \in F x$, for $F x$ is compact, a contradiction. If $F x \subset M \backslash X$, then there exists a $z \in F x$ such that $d(x, z)=$ $d(x, F x)$ since $F x$ is compact. Therefore, $d(z, x)=d(x, F x) \leq d(y, F x) \leq d(y, z)=$ $d(z, y)$ for all $y \in X$, that is, $d(z, x)=d(z, X)$. Hence $x \in \partial X$, and by (2), $F x \cap X \neq \varnothing$, another contradiction.

Clearly, $(4) \Rightarrow(3) \Rightarrow(2)$. This completes our proof.

REMARK. Corollary 2.1.1(4) is comparable to a well-known result that, if $X$ is a compact acyclic ANR and $F: X \rightarrow 2^{X} \backslash\{\phi\}$ u.s.c. with $F x$ acyclic for each $x \in X$, then $F$ has a fixed point (Eilenberg and Montgomery [3]). However, the following example of Gwinner [6, p. 575] shows that the lower semicontinuity of $F$ in Corollary 2.1.1(1) is not dispensable.

EXAMPLE. Let $X=\{(c, 0) \mid c \in[0,1]\} \subset R^{2}=M$. Define $F: X \rightarrow 2^{M} \backslash\{\phi\}$ by

$$
\begin{aligned}
& F(c, 0)=\operatorname{co}\{(1,1),(1,2)\} \quad \text { if } 0 \leq c<1, \\
& F(1,0)=\operatorname{co}\{(0,0),(1,1),(1,2)\} .
\end{aligned}
$$

Then $F$ satisfies the hypothesis of Corollary 2.1.1(1) except the l.s.c. of $F$. However, $F$ has no fixed point. 
COROLlARY 2.1.2. Let $X$ be a compact acyclic finite-dimensional ANR subset of a metric space, and $f: X \rightarrow M$ continuous such that for all $x \in X$, the set $\{y \in X \mid d(x, f x)>d(y, f x)\}$ is contractible or empty.

Then (i) $\sim$ (vi) of Theorem 2.1 with $F=f$ hold. Further, $f$ has a fixed point if one of the following conditions holds.

(1) For each $x \in X$ with $x \neq f x$, there exists a $y \in X$ such that $d(x, f x)>$ $d(y, f x)$.

(2) $f(\partial X) \subset X$.

(3) $f(X) \subset X$.

REMARK. Case (2) in Corollary 2.1.2 is due to McClendon [7, Theorem 3.6]. The case (3) follows from the well-known fact that every compact acyclic ANR has the fixed point property.

From Theorem 1.2, we have

THEOREM 2.2. Let $X$ be a compact acyclic lc subspace of a metric space $(M, d)$, and $F: X \rightarrow 2^{M} \backslash\{\phi\}$ a multifunction satisfying

(a) $x \mapsto d(x, F x)$ is continuous on $X$,

(b) $(x, y) \mapsto d(x, F y)$ is l.s.c.,

(c) for each $x \in X, y \mapsto d(x, F y)$ is u.s.c., and

(d) $\{x \in X \mid d(x, F y) \leq t\}$ is acyclic or empty for all $y \in X$ and $t \in R$.

Then the statements $(\mathrm{i}) \sim(\mathrm{vi})$ of Theorem 2.1 hold.

ProOF. Define $p: X \times X \rightarrow R$ by $p(x, y)=d(x, F y)$. Then (i) follows from Theorem 1.2. The equivalency of (i) $\sim$ (vi) is the same as Theorem 2.1 .

REMARK. If $F y \subset X$ and $F y$ is closed, then (d) implies that $F y$ is acyclic, for $F y=\{x \in X \mid d(x, F y) \leq 0\}$.

COROLLARY 2.2.1. Let $X$ be a compact acyclic lc subspace of a metric space $M$, and $F: X \rightarrow 2^{M} \backslash\{\phi\}$ continuous compact-valued such that $\{x \in X \mid d(x, F y) \leq$ $t\}$ is acyclic or empty for all $y \in X$ and $t \in R$. Then the conclusion of Corollary 2.1.1 holds.

PROOF. For a continuous compact-valued multifunction $F$, the conditions (a), (b), and (c) of Theorem 2.2 hold, by Theorems 1 and 2 of Berge [2, p. 121].

REMARK. The case (4) is a consequence of Begle's well-known theorem in [1]: If $X$ is a compact acyclic lc space and $\mathscr{C}(X)$ the class of all closed acyclic subsets of $X$, then any u.s.c. $F: X \rightarrow \mathscr{C}(X)$ has a fixed point. However, as in the remark following Corollary 2.1.1, Gwinner's example shows that the lower semicontinuity of $F$ in Corollary 2.2.1 (1) is not dispensable.

COROLLARY 2.2.2. Let $X$ be a compact acyclic lc subspace of a metric space $M$, and $f: X \rightarrow M$ continuous such that $\{x \in X \mid d(x, f y) \leq t\}$ is acyclic for all $y \in X$ and $t \in R$. Then the conclusion of Corollary 2.1.2 holds.

REMARK. Corollary 2.2.2 (i) and the case (2) are due to McClendon [7, Theorem 3.7 and Corollary 3.8]. Corollary 2.2.2 (i) generalizes Fan [4, Theorem 2]. For the case (3), if $f$ is a self-map of $X$, then the condition on $\{x \in X \mid d(x, f y) \leq t\}$ is superfluous, by Begle's theorem mentioned above.

From Theorem 1.3, we have the following theorem as a final conclusion. The proof is parallel and some corollaries of it also can be obtained as in Theorems 2.1 and 2.2 , so we omit them. 
THEOREM 2.3. Let $X$ be a compact acyclic lc subspace of a metric space $(M, d)$, and $F: X \rightarrow 2^{M} \backslash\{\phi\}$ a multifunction satisfying

(a) $d(x, F y)$ is continuous on $X \times X$,

(b) $\{x \mid d(x, F y)=\inf \{d(z, F y) \mid z \in X\}\}$ is acyclic for each $y \in X$.

Then statements $(\mathrm{i}) \sim(\mathrm{vi})$ of Theorem 2.1 hold.

ACKNOWLEDGEMENT. The author thanks the referee for his helpful advice.

\section{REFERENCES}

1. E. G. Begle, A fixed point theorem, Ann. of Math. (2) 51 (1950), 544-550.

2. C. Berge, Espaces topologiques, Dunod, Paris, 1959.

3. S. Eilenberg and D. Montgomery, Fixed point theorems for multi-valued transformations, Amer. J. Math. 68 (1946), 214-222.

4. K. Fan, Extensions of two fixed point theorems of F. E. Browder, Math. Z. 112 (1969), 234-240.

5. __ A minimax inequality and applications, (O. Shisha, ed.), Inequalities III, Proc. 3rd Sympos. on Inequalities, Academic Press, New York, 1972, pp. 103-113.

6. J. Gwinner, On fixed points and variational inequalities - a circular tour, Nonlinear Analysis 5 (1981), 565-583.

7. J. F. McClendon, Minimax and variational inequalities for compact spaces, Proc. Amer. Math. Soc. 89 (1983), 717-721.

8. R. Thompson, Fixed point indices in locally convex spaces, Adv. in Math. 14 (1974), 73-91.

9. T. Van der Walt, Fixed and almost fixed points, Math. Centrum, Amsterdam, 1963.

Department of Mathematics, Seoul National University and The MatheMATICAL SCIENCES RESEARCh INSTITUTE OF KOREA, SEOUL 151-742, Korea 